\title{
Clinical Benefit of Hepatic Arterial Infusion Concurrent Chemoradiotherapy in Locally Advanced Hepatocellular Carcinoma: A Propensity Score Matching Analysis
}

\author{
Hong In Yoon, MD ${ }^{1,2}$ \\ Ki Jun Song, $P h D^{3}$ \\ Ik Jae Lee, MD, $\mathrm{PhD} 1,4,5$ \\ Do Young Kim, MD, PhD ${ }^{4,5,6}$ \\ Kwang-Hyub Han, MD ${ }^{4,5,6}$ \\ Jinsil Seong, MD, PhD ${ }^{1,4,5}$
}

\section{${ }^{1}$ Department of Radiation Oncology, Yonsei Cancer Center, Yonsei University College of Medicine, Seoul, ${ }^{2}$ Department of Pharmacology, Brain Korea 21 Plus Project for Medical Science, Yonsei University College of Medicine, Seoul, \\ ${ }^{3}$ Department of Biostatics, Yonsei University College of Medicine, Seoul, \\ ${ }^{4}$ Yonsei Liver Cancer Special Clinic, Yonsei University College of Medicine, Seoul, ${ }^{5}$ Korean Liver Cancer Study Group, Seoul, ${ }^{6}$ Department of Internal Medicine, Yonsei University College of Medicine, Seoul, Korea}

Correspondence: Jinsil Seong, MD, PhD Department of Radiation Oncology, Yonsei Cancer Center, Yonsei University Health System, 50-1 Yonsei-ro, Seodaemun-gu, Seoul 03722, Korea

Tel: 82-2-2228-8095

Fax: 82-2-2227-7823

E-mail: jsseong@yuhs.ac

Received September 29, 2014

Accepted December 18, 2014

Published Online March 5, 2015

${ }^{*}$ This work has been presented at the 7th Annual Conference of the International Liver Cancer Association (ILCA), in Washington, D.C., USA, September 13-15, 2013.

\section{Purpose}

The aim of this study was to evaluate whether hepatic arterial infusion concurrent chemoradiotherapy (CCRT) could improve overall survival (OS) in patients with locally advanced hepatocellular carcinoma (LAHCC).

\section{Materials and Methods}

Two databases were reviewed from Yonsei Cancer Center (YCC) and Korean Liver Cancer Study Group (KLCSG) nationwide multi-center hepatocellular carcinoma (HCC) cohort. The CCRT group included 106 patients, with stage III-IV, Child-Pugh classification A, Eastern Cooperative Oncology Group performance status 0 or 1 , who underwent definitive CCRT as the initial treatment at YCC. We used propensity score matching to adjust for seven clinical factors, including age, tumor size, TNM stage by the Liver Cancer Study Group of Japan, T stage, Barcelona Clinic Liver Cancer (BCLC) staging system, etiology of HCC, and portal vein invasion, which all differed significantly in the two databases. From the KLCSG cohort enrolled at 32 institutions, 106 patients for the non-CCRT group were defined.

\section{Results}

After propensity score matching, all patient characteristics were balanced between the two groups. The CCRT group had better OS (median, 11.4) than the non-CCRT group (6.6 months, $p=0.02$ ). In multivariate analyses for all patients, CCRT (hazard ratio [HR], 1.48; 95\% confidence interval [Cl], 1.11 to $1.97 ; p=0.007)$, tumor size (HR, 1.08; 95\% Cl, 1.04 to $1.12 ; p<0.001)$, and BCLC stage (HR, $0.54 ; 95 \% \mathrm{Cl}, 0.36$ to $0.8 ; \mathrm{p}=0.003$ ) were independent prognostic factors for OS.

\section{Conclusion}

CCRT showed better OS for LAHCC patients. In LAHCC patients with a good performance and normal liver function, CCRT could be a feasible treatment option. All of these findings need to be validated in prospective clinical trials.

\section{Introduction}

Hepatocellular carcinoma (HCC) is listed as the sixth most common cancer type and third most common cause of can-

\section{Key words}

Hepatocellular carcinoma, Radiotherapy, Propensity score cer-related deaths [1]. According to the Barcelona Clinic Liver Cancer (BCLC) staging system, potential curative therapies can be applied to early stages of the disease [2]. However, the majority of HCC patients, who are not in early stage at the time of the first diagnosis only receive palliative or con- 
servative treatments.

In cancer treatment, a multimodality approach by a multidisciplinary team has been the key principle, using surgery and radiotherapy (RT) for local and loco-regional sites and chemotherapy for systemic diseases $[3,4]$. This principle has proved to be effective in most cancers $[5,6]$. Particularly, in locally advanced cancers, it can induce down-staging to resectable status that can be followed by radical resection. Unfortunately, a multimodality approach involving RT was not successful for HCC some decades ago [7]. Whole liver irradiation with radiation dose less than the tumoricidal level could be one of the major reasons for the failure in a multimodality approach involving RT.

A recent development of RT technologies allows the delivery of high doses safely and effectively to localized tumor volume and has shown a potential to improve survival without severe radiation-related toxicity [8-10]. In the National Comprehensive Cancer Network (NCCN) guidelines for $\mathrm{HCC}$, external beam RT is recommended in patients with unresectable disease, who are not transplant candidates and in patients with inoperable local disease despite the evidence of being category 2B [11]. In our institution, localized concurrent chemoradiotherapy (CCRT) for HCC consisted of local RT and hepatic arterial infusion chemotherapy (HAIC) of 5-fluorouracil (5-FU); CCRT was performed to enhance the effect of local RT and to reduce the risk of intrahepatic metastasis. In several studies at our institution, CCRT showed favorable outcomes $[12,13]$. Several studies from other institutions reporting about CCRT with HAIC have previously demonstrated fine treatment outcomes $[8,14]$. However, due to the absence of sufficient evidence from phase III randomized control trials, the American Association for the Study of Liver Diseases and the European Association for the Study of the Liver guidelines do not recommend RT as a treatment option [2].

In Korea, Korean Liver Cancer Study Group (KLCSG) established a randomly enrolled nationwide multi-center HCC cohort between 2003 and 2005 from 32 hospitals in an effort to generate an optimal management strategy of HCC [15]. The cohort provides unbiased information regarding clinical characteristics, treatment patterns, and overall survival of HCC patients in Korea. However, most patients who were enrolled in the KLCSG cohort received other monotherapies, rather than multimodal therapy, such as CCRT as the first treatment following the diagnosis of locally advanced HCC. To generate and verify a hypothesis to help implement a randomized control trial, we utilized the KLCSG cohort as the control group for comparisons between CCRT and nonCCRT groups, using propensity score matching. In this study, we evaluated whether CCRT could improve survival for locally advanced HCC compared to other treatments, using propensity score matching.

\section{Materials and Methods}

\section{Study design}

The study was performed and reported in accordance to the recommendations of STROBE statement [16]. We reviewed two databases: the Yonsei Cancer Center (YCC) and KLCSG nationwide multi-center HCC cohort. This study received approval from the internal review boards of participating institutions (IRB No. 4-2013-0897). We identified 829 patients who underwent RT for locally advanced HCC at YCC between 1992 and 2008. Patients were excluded if any of the following conditions were met: (1) stage I-II disease, (2) extrahepatic metastasis (not including regional lymph node involvement), (3) Child-Pugh classification B or C, (4) CCRT not the first treatment, (5) initially prescribed with insufficient irradiated dose for definitive RT ( $<40 \mathrm{~Gy}$ ), (6) Eastern Cooperative Oncology Group performance status (ECOG PS) $>2$, (7) a history of RT to the abdominal area, or (8) RT alone or RT with intravenous chemotherapy. After exclusions, a total of 106 patients were diagnosed between 1999 and 2008, and patients who underwent hepatic arterial infusion CCRT definitively as the initial treatment were included in the CCRT group.

The KLCSG nationwide multi-center cohort included 4,522 patients from 2003 to 2005 at 32 Korean institutions. Among them, 77 patients were excluded due to false information regarding the first treatment. Next, 751 locally advanced HCC patients who underwent other initial treatments (except RT) were identified by excluding patients who (1) had stage I-II cancer, (2) were given M1 classification, (3) were given Child-Pugh classification B or C, (4) received RT, (5) had missing information for CP class or treatment type or date, or (6) had false information regarding the treatment aim or diagnosis date. The KLCSG cohort included information regarding age, gender, method of diagnosis, date of diagnosis, date of death, Child-Pugh classification, TNM stage by Liver Cancer Study Group of Japan (LCSGJ), tumor size, growth pattern of tumor, tumor invasion into major vessels, $\mathrm{T}$ stage, $\mathrm{N}$ stage, $\mathrm{M}$ stage, BCLC staging, the etiology of HCC, type of initial treatment modality, and date of initial treatment. Among the 751 patients, we assigned 106 patients receiving other monotherapies into the non-CCRT group by performing 1 to 1 propensity score matching with the CCRT group.

Staging was determined according to the TNM staging system of the LCSGJ [17] and BCLC staging system [2]. HCC was diagnosed depending on the practice guidelines for diagnosis and treatment of HCC in KLCSG [18]. HCC was defined as pathologic confirmation of HCC or compatible radiological findings with serum alpha-fetoprotein (AFP) 
Table 1. Patient characteristics

\begin{tabular}{|c|c|c|c|c|c|c|}
\hline \multicolumn{2}{|l|}{ Variable } & $\begin{array}{c}\text { CCRT } \\
(n=106)\end{array}$ & $\begin{array}{l}\text { KLCSG cohort } \\
\qquad(n=751)\end{array}$ & p-value & $\begin{array}{c}\text { Non-CCRT } \\
(n=106)\end{array}$ & p-value \\
\hline \multicolumn{2}{|l|}{ Median age (range, yr) } & $52(11-79)$ & $57(8-83)$ & $<0.001$ & $52(8-74)$ & 0.99 \\
\hline \multicolumn{2}{|c|}{ Median tumor size (range, $\mathrm{cm}$ ) } & $10(2-17)$ & $6(1-21)$ & $<0.001$ & $10(2-21)$ & 0.53 \\
\hline \multirow[t]{2}{*}{ Growth pattern } & Single & $31(29.2)$ & $269(35.8)$ & 0.18 & $31(29.2)$ & $>0.99$ \\
\hline & Multiple & $75(70.8)$ & $482(64.2)$ & & $75(70.8)$ & \\
\hline \multirow[t]{2}{*}{ Sex } & Male & $90(84.9)$ & $656(87.4)$ & 0.48 & $90(84.9)$ & $>0.99$ \\
\hline & Female & $16(15.1)$ & $95(12.6)$ & & $16(15.1)$ & \\
\hline Child-Pugh classification & $\mathrm{A}$ & $106(100)$ & $751(100)$ & $>0.99$ & $106(100)$ & $>0.99$ \\
\hline \multirow[t]{2}{*}{ TNM stage by LCSGJ } & III & $47(44.3)$ & 585 (77.9) & $<0.001$ & $47(44.3)$ & $>0.99$ \\
\hline & IV-A & $59(55.7)$ & $166(22.1)$ & & $59(55.7)$ & \\
\hline \multirow[t]{4}{*}{ T stage } & $\mathrm{T} 1$ & 0 & $2(0.3)$ & $<0.001$ & 0 & 0.89 \\
\hline & $\mathrm{T} 2$ & 0 & $12(1.6)$ & & 0 & \\
\hline & $\mathrm{T} 3$ & $48(45.3)$ & $606(80.7)$ & & $49(46.2)$ & \\
\hline & $\mathrm{T} 4$ & $58(54.7)$ & $131(17.4)$ & & $57(53.8)$ & \\
\hline \multirow[t]{2}{*}{$\mathrm{N}$ stage } & N0 & 97 (91.5) & $706(94.0)$ & 0.32 & $98(92.5)$ & 0.8 \\
\hline & N1 & $9(8.5)$ & $45(6.0)$ & & $8(7.5)$ & \\
\hline \multirow[t]{2}{*}{ Portal vein invasion } & Yes & $85(80.2)$ & $343(45.7)$ & $<0.001$ & $85(80.2)$ & $>0.99$ \\
\hline & No & $21(19.8)$ & $408(54.3)$ & & $21(19.8)$ & \\
\hline \multirow[t]{2}{*}{ Hepatic vein invasion } & Yes & $6(5.7)$ & $77(10.3)$ & 0.13 & $13(12.3)$ & 0.09 \\
\hline & No & $100(94.3)$ & $671(89.7)$ & & $93(87.7)$ & \\
\hline \multirow[t]{3}{*}{ BCLC staging system } & $\mathrm{A}$ & 0 & $93(12.4)$ & $<0.001$ & 0 & 0.85 \\
\hline & B & $18(17.0)$ & 247 (32.9) & & $17(16.0)$ & \\
\hline & $\mathrm{C}$ & $88(83.0)$ & $411(54.7)$ & & $89(84.0)$ & \\
\hline \multirow{5}{*}{$\begin{array}{l}\text { The etiology of } \\
\text { hepatocellular carcinoma }\end{array}$} & HBV & $83(78.3)$ & $487(64.8)$ & 0.01 & $84(79.2)$ & 0.79 \\
\hline & $\mathrm{HCV}$ & $6(5.7)$ & $76(10.1)$ & & $3(2.8)$ & \\
\hline & NBNC and alcohol & $13(12.2)$ & $77(10.3)$ & & $15(14.2)$ & \\
\hline & $\mathrm{HBV}$ and $\mathrm{HCV}$ & 0 & $25(3.3)$ & & 0 & \\
\hline & Unknown & $4(3.8)$ & $86(11.5)$ & & $4(3.8)$ & \\
\hline
\end{tabular}

Values are presented as number (\%) unless otherwise indicated. CCRT, concurrent chemoradiotherapy; KLCSG, Korean Liver Cancer Study Group; LCSGJ, Liver Cancer Study Group of Japan; TNM, tumor, node, metastasis; BCLC, Barcelona Clinic Liver Cancer; HBV, hepatitis B virus; HCV, hepatitis C virus; NBNC, non-HBV or non-HCV hepatitis.

level > $400 \mathrm{IU} / \mathrm{mL}$. If AFP levels were $\leq 400 \mathrm{IU} / \mathrm{mL}$, HCC was diagnosed by radiological findings using at least two imaging modalities.

\section{1) Concurrent chemoradiotherapy}

In the CCRT group, the total radiation dose was a median of $45 \mathrm{~Gy}$ (range, 42.4 to $59.4 \mathrm{~Gy}$ ) in 25 fractions (range, 16 to 33). We defined and delineated the gross tumor volume, clinical target volume (CTV), and planning target volume (PTV) to determine the prescription dose as described previously [10]. PTV was defined as CTV surrounded by an additional 5-10 mm margin for setup error and internal organ motion. We irradiated PTV with a surrounding margin of $1 \mathrm{~cm}$ in a 3-dimensional conformal radiotherapy (3D-CRT) in accor- dance to the dose prescription guidelines [10]. The radiation dose for a target volume depended on the functional reserve of the liver and was chosen in a range acceptable to the dose prescription guideline at each V50\% (the normal liver volume irradiated with more than one-half of the prescription dose) category. For image-guided intensity-modulated RT before April 2007, dose prescription was also determined similar to 3D-CRT. After April 2007, the prescribed radiation dose was determined according to dose-volume parameters of the effective irradiated liver volume [10]. Thus, we limited the mean liver dose to $23 \mathrm{~Gy}$ and the maximal dose to 42.24 Gy for the duodenum, stomach, small bowel, and spinal cord. Concurrent continuous-infusion hepatic arterial 5-FU, using a percutaneous hepatic arterial catheter inserted via hepatic arterial angiography, was delivered at a dose of 500 


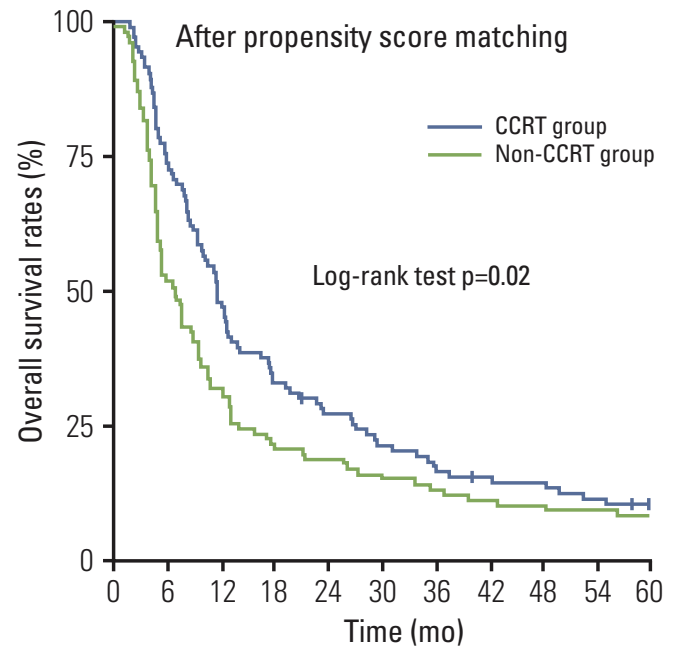

No. at risk

$\begin{array}{llllllllllll}\text { Non-CCRT } & 106 & 55 & 34 & 22 & 20 & 16 & 14 & 12 & 11 & 10 & 6\end{array}$

$\begin{array}{llllllllllll}\text { CCRT } & 106 & 77 & 50 & 35 & 28 & 22 & 17 & 15 & 14 & 10 & 4\end{array}$

Fig. 1. Kaplan-Meier curve of overall survival (OS) according to the two treatment groups. Kaplan-Meier curve depicted that concurrent chemoradiotherapy (CCRT) (median OS, 11.4 months; 2-year OS, 27.3\%; 5-year OS, $10.4 \%$ ) significantly improved OS compared to the non-CCRT group (median OS, 6.6 months; 2-year OS, $18.9 \%$; 5-year OS, 8.5\%; $\mathrm{p}=0.02$ ).

$\mathrm{mg} /$ day during the first and last weeks of RT [12]. Most patients received HAIC with 5 -FU (at a dose of $500 \mathrm{mg} / \mathrm{m}^{2}$ for 5 hours on days 1-3) and cisplatin (at a dose of $60 \mathrm{mg} / \mathrm{m}^{2}$ for 2 hours on day 2) for three to 12 cycles in accordance to the treatment response after CCRT. In cases of multiple tumors, the primary and adjacent tumors were irradiated. In 12 patients $(16.0 \%)$ among the 75 multiple tumor patients, additional tumors outside of the RT field were treated using a transcatheter arterial chemoembolization (TACE) [13] or transcatheter arterial chemoinfusion (TACI).

\section{2) Treatment modality in non-CCRT group}

In the non-CCRT group, 12 patients were treated with surgical resection $(11.3 \%)$, one $(0.9 \%)$ with local ablation therapy, $78(73.6 \%)$ with TACE, 13 patients $(12.3 \%)$ with local chemotherapy such as TACI or transcatheter arterial lipiodol-chemoinfusion, and two $(1.9 \%)$ with systemic chemotherapy. The KLCSG database only included information of the treatment type; therefore, detailed treatment methods are unknown.

\section{3) End points}

The overall actuarial survival (OS) was calculated from the RT start date to the date of death or last follow-up for the CCRT group and from the treatment date to the date of death or last contact for non-CCRT group. We compared the OS rates between the two groups.

\section{4) Subgroup analyses}

In additional subgroups according to prognostic factors, we compared the OS rate between the CCRT and non-CCRT groups. We considered tumor size $\geq 5 \mathrm{~cm}$, treatment period, BCLC stage C, portal vein invasion (PVI), or major (lobar or main) PVI as prognostic factors to determine each subgroup.

\section{5) Statistical considerations}

As described in Table 1, the CCRT group and KLCSG cohort differed significantly in several clinical factors, including age, tumor size, TNM stage by LCSGJ, T stage, PVI, BCLC stage, and the etiology of HCC. Therefore, we performed propensity score matching to adjust for seven potentially significant patient characteristics that differed significantly between the CCRT group and the KLCSG cohort (age, tumor size, TNM stage by the LCSGJ, T stage, BCLC stage, the etiology of HCC, and PVI). Using multivariate logistic regression analyses to make weighted probabilities, propensity scores were calculated to determine the effect of characteristics on the treatment modality. Propensity score matching determined that the non-CCRT group of 106 patients who underwent other monotherapies from the KLCSG database matched with the 106 patients of the CCRT group from the YCC database. To appraise the degree of balancing, we compared clinical and prognostic factors between the two groups using Pearson's chi-square tests or Fisher exact tests for nominal variables, and Mann-Whitney U tests for continuous variables. We calculated OS rates using the Kaplan-Meier method and log-rank tests to assess the survival differences. We performed univariate and multivariate analyses to evaluate all prognostic factors influencing OS using Cox's regression model. We performed a multivariate analysis using backwards elimination to stay in the model. The hazard ratios (HR) are given with 95\% confidence intervals $(95 \% \mathrm{CI})$. We considered p-values less than 0.05 to be statistically significant. SPSS ver. 20.0 (IBM Co., Armonk, NY) was used for all analyses. 
Table 2. Prognostic factors for overall survival based on Cox proportional-hazards model $(n=212)$

\begin{tabular}{|c|c|c|c|c|}
\hline \multirow{2}{*}{ Variable } & \multicolumn{2}{|c|}{ Univariate analyses } & \multicolumn{2}{|c|}{ Multivariate analyses } \\
\hline & HR $(95 \%$ CI $)$ & p-value & HR $(95 \% \mathrm{CI})$ & p-value \\
\hline Age (yr) & $1(0.99-1.01)$ & 0.63 & - & - \\
\hline Tumor size $(\mathrm{cm})$ & $1.08(1.03-1.12)$ & $<0.001$ & $1.08(1.04-1.12)$ & $<0.001$ \\
\hline Sex (male vs. female) & $1.17(0.8-1.72)$ & 0.42 & - & - \\
\hline Growth pattern (single vs. multiple) & $0.84(0.62-1.15)$ & 0.29 & - & - \\
\hline $\begin{array}{l}\text { Treatment period } \\
(<2003,2003-2005,>2005)\end{array}$ & $\begin{array}{c}\text { Reference } \\
1.21(0.77-1.9) \\
1.23(0.85-1.76)\end{array}$ & 0.54 & - & - \\
\hline TNM stage by LCSGJ (III vs. IV-A) & $0.6(0.45-0.8)$ & 0.001 & - & - \\
\hline T stage (T3 vs. T4) & $0.6(0.45-0.8)$ & 0.001 & - & - \\
\hline $\mathrm{N}$ stage (N0 vs. N1) & $1.03(0.61-1.75)$ & 0.9 & - & - \\
\hline Portal vein invasion (no vs. yes) & $0.57(0.39-0.82)$ & 0.003 & - & - \\
\hline BCLC staging system (B vs. C) & $0.54(0.36-0.81)$ & 0.003 & $0.54(0.36-0.8)$ & 0.003 \\
\hline CCRT (no vs. yes) & $1.41(1.06-1.87)$ & 0.02 & $1.48(1.11-1.97)$ & 0.007 \\
\hline
\end{tabular}

HR, hazard ratio; CI, confidence interval; TNM, tumor, node, metastasis; LCSGJ, Liver Cancer Study Group of Japan; BCLC, Barcelona Clinic Liver Cancer; CCRT, concurrent chemoradiotherapy.

\section{Results}

\section{Patient characteristics after propensity score matching}

The patient characteristics of the two groups after propensity score matching are listed in Table 1. Before propensity score matching, there were significant differences in patient characteristics, including age $(\mathrm{p}<0.001)$, tumor size $(\mathrm{p}<0.001)$, TNM stage $(p<0.001)$, T stage $(p<0.001)$, PVI $(p<0.001)$, BCLC staging $(p<0.001)$ and the etiology of HCC $(p=0.01)$ between the CCRT group and KLCSG cohort. After propensity score matching, Table 1 demonstrates that all clinical and prognostic factors were balanced between the CCRT and non-CCRT groups.

\section{Comparison of overall survival rates between the two treatment groups}

The median follow-up period for surviving patients was 63 months for the CCRT group and 90 months for the nonCCRT group. After propensity score matching analyses, Kaplan-Meier curves depicted that the CCRT group (median OS, 11.4 months; 5-year OS, 10.4\%) had significantly better OS than the non-CCRT group (median OS, 6.6 months; 5-year OS, 8.5\%; $\mathrm{p}=0.02$ ) (Fig. 1).

\section{Prognostic factors analyses}

We performed prognostic factor analyses using a Cox regression model for 212 patients in both groups (Table 2). Univariate analyses showed that tumor size (HR, 1.08; 95\% CI, 1.03 to $1.12 ; \mathrm{p}<0.001$ ), TNM stage by LCSGJ (III vs. IV-A; HR, 0.6; 95\% CI, 0.45 to $0.8 ; \mathrm{p}=0.001$ ), T stage (T3 vs. T4; HR, 0.6; 95\% CI, 0.45 to 0.8; $\mathrm{p}=0.001$ ), PVI (no vs. yes; HR, $0.57 ; 95 \% \mathrm{CI}, 0.39$ to $0.82 ; \mathrm{p}=0.003)$, BCLC staging system (B vs. $\mathrm{C} ; \mathrm{HR}, 0.54 ; 95 \% \mathrm{CI}, 0.36$ to $0.81 ; \mathrm{p}=0.003$ ) and CCRT (no vs. yes; HR, $1.41 ; 95 \% \mathrm{CI}, 1.06$ to $1.87 ; \mathrm{p}=0.02$ ) were significantly associated with OS. The treatment period did not affect OS. In multivariate analyses using all prognostic factors, CCRT (no vs. yes; HR, 1.48; 95\% CI, 1.11 to 1.97 ; $\mathrm{p}=0.007)$, tumor size (HR, 1.08; 95\% CI, 1.04 to $1.12 ; \mathrm{p}<0.001)$ and BCLC staging system (B vs. C; HR, 0.54; 95\% CI, 0.36 to $0.8 ; \mathrm{p}=0.003$ ) were independent prognostic factors for $\mathrm{OS}$.

\section{Subgroup analyses}

Because CCRT showed statistically significant differences after prognostic factor analyses, we evaluated the survival benefit of CCRT in several subgroups. We assessed whether CCRT influenced OS as a prognostic factor in each subgroup using a Cox regression model (Fig. 2). CCRT was a statistically significant factor for OS, and the CCRT group showed significantly better OS than the non-CCRT group in subgroups with a tumor size $\geq 5 \mathrm{~cm}$ (no vs. yes; HR, 1.75; 95\% CI, 1.3 to 2.37; $\mathrm{p}<0.001$ ). In the subgroup of patients who underwent treatment from 2003 to 2005, CCRT was also a significant factor for OS (no vs. yes; $\mathrm{HR}, 1.91 ; 95 \% \mathrm{CI}, 1.15$ to 3.17; $\mathrm{p}=0.01$ ). CCRT-treated patients showed longer OS with a statistical significance in the BCLC C subgroup (no vs. yes; 


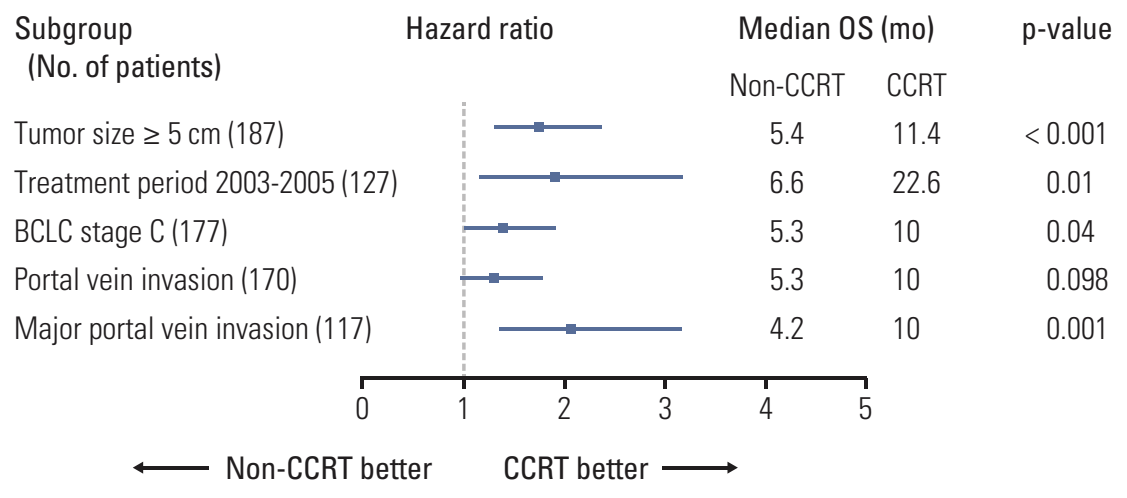

Fig. 2. The difference in overall survival (OS) between the two treatment groups in each subgroup. CCRT, concurrent chemoradiotherapy; BCLC, Barcelona Clinic Liver Cancer.

HR, $0.54 ; 95 \% \mathrm{CI}, 0.36$ to $0.8 ; \mathrm{p}=0.04)$. In the PVI subgroup, CCRT-treated patients showed a longer median survival, although this was not statistically significant. However, CCRT was significantly related to OS in the major PVI subgroup (no vs. yes; HR, 2.07; 95\% CI, 1.35 to 3.16; $\mathrm{p}=0.001$ ).

\section{Discussion}

In this study, all clinical and prognostic factors were balanced between the two groups after matching. The CCRT group showed a significantly better OS than the non-CCRT group. CCRT, tumor size, and BCLC staging system were independent prognostic factors for OS on multivariate analyses. CCRT was also a significant factor for OS in each subgroup of patients receiving treatment from 2003 to 2005, having tumor size $\geq 5 \mathrm{~cm}$, BCLC stage $C$, or showing major PVI.

However, special considerations are required to interpret the findings of this study due to the following limitations. First, this was a retrospective study, which was an unavoidable weakness. However, it is believed that a propensity score analytic approach could minimize this limitation. Second, we did not include patients receiving sorafenib, as most patients were treated for HCC before the era of sorafenib. Although our results show that the OS of CCRT group was comparable to that reported for sorafenib [19,20], we suggest that prospective randomized trials comparing CCRT to sorafenib are be necessary. Third, two different databases were utilized for this study. In 2003, for the first time, the KLCSG and the National Cancer Center (NCC) of Korea produced a clinical practice guideline in Korea. Since
2003, all Korean institutions, including our own, have complied with the KLCSG guidelines, and most of the research publications from our institution indicate that we have diagnosed HCC based on the KLCSG guidelines [10,13]. Despite this limitation, we believe that the characteristics of patients included in the two databases should not be very different. However, since the treatment period of these two databases was different, we analyzed the effect of the treatment period in order to adjust for such differences. The treatment period used for the KLCSG database was from 2003 to 2005. Thus, we classified the treatment period as starting from 2003 until 2005. In the analyses of prognostic factors, the treatment period was not a significant factor for OS (Table 2). In the subgroup analysis of patients treated between 2003 and 2005, the CCRT group showed a significantly higher OS (Fig. 2). Due to the different databases, it is also possible that those in the non-CCRT group may have been in more advanced stages of HCC. However, given that the CCRT group showed a tumor growth pattern that was similar to the non-CCRT group, albeit with more major PVI (CCRT, 80.2\%; non-CCRT, 30.2\%; p < 0.001), we do not believe that the non-CCRT group patients in this study were of a more advanced stage at the time of diagnosis. Based on this, we believe that the utilization of two databases should not have affected our findings or conclusions. Fourth, we could not use AFP levels and performance status of patients for propensity score matching analysis as the KLCSG databases did not include information regarding the AFP level and performance status. In future studies, AFP levels and performance status need to be considered as they could be associated with overall survival. Fifth, at least a certain portion of patients in the CCRT group might have been highly selected patients, which might have in turn influenced the treatment outcome, given that all patients in the CCRT group 
were treated at the tertiary referral hospital only. Thus, further prospective randomized clinical trials are necessary to prove our findings.

A multimodality treatment, such as CCRT, has been repeatedly proven to be effective in advanced stages of several cancers. However, the survival benefit of RT or chemotherapy alone as well as CCRT for HCC has not been fully elucidated. Nevertheless, several combined treatments, such as TACE plus RT, or CCRT with HAIC have been utilized in attempts to improve the oncologic outcome for HCC. Therefore, several studies regarding a combination of RT and TACE have reported favorable response rates and overall survival for HCC with PVI [21,22]. Furthermore, some studies investigating the combination of RT with concurrent hepatic artery chemotherapy have also demonstrated a favorable effect on overall survival, with a median survival of 11-15.2 months for unresectable primary hepatobiliary cancer $[14,23]$. These results are comparable to the results of this study. The purpose of using CCRT with HAIC was to enhance the local radio-therapeutic effect and to reduce intrahepatic HCC spread. As such, although every treatment for HCC is controversial, we anticipated that concurrently combining HAIC with RT would produce a synergistic therapeutic effect. Based on these concepts of a multimodality approach, our group previously reported that CCRT improved response rates and survival for locally advanced HCC with PVI [12]. In a more recent study, it was reported that CCRT or a combination of RT and TACE produced an excellent intrahepatic control as well as prolonged survival [24]. The median OS was significantly longer in the combined RT and TACE group (15.3 months) and CCRT group (12.8 months) than in TACE alone (7.5 months) and HAIC alone (8.2 months) for huge HCC larger than $10 \mathrm{~cm}$. In the subgroup analysis of this study, the CCRT group had significantly better OS compared to the non-CCRT group for locally advanced HCC with major PVI. Our findings suggest that CCRT could be a feasible treatment option for HCC with major PVI.

Furthermore, CCRT can potentially cure selected locally advanced patients by inducing down-staging and conversion to resectable status. In a recent study, our group reported that CCRT increased resectability by down-staging tumors [25]. Tumor resectability was assessed by liver surgeons and radiologists after completing CCRT, using all computed tomography or magnetic resonance imaging scans. Curative resection was performed for patients showing radiologic findings that all gross lesions could be resected with a clear margin. Among a total of 243 patients who underwent CCRT, 41 patients (16.9\%) received curative resection. Downstaging of T classification after CCRT was observed in 32 (78.0\%). On Kaplan-Meier survival curves, 41 patients who received curative resection showed significantly higher
5 -year OS $(49.6 \%)$ than the others $(0 \%, \mathrm{p}<0.001)$. In this study, 12 patients $(11.3 \%)$ underwent curative resection, and $10(83.3 \%)$ of them showed down-staging of T classification. In the CCRT group, the 12 patients who received a curative resection showed a significantly better 5-year OS (72.7\%) than the other patients $(2.5 \%, \mathrm{p}<0.001)$ and showed a significantly higher 5-year OS than patients who underwent resection as the first line of treatment in the non-CCRT group (16.7\%, $\mathrm{p}=0.002$ ). We consider CCRT as a potential contributor to a better treatment outcome in locally advanced HCC patients without extra-hepatic visceral metastasis. However, a well-designed further prospective study would be needed to prove a down-staging effect of CCRT for locally advanced HCC.

\section{Conclusion}

In conclusion, this study used propensity score matching analyses to show that CCRT was significantly associated with better OS for locally advanced HCC. We believe that in patients with good performance status and normal liver function, CCRT could be a feasible treatment option for locally advanced HCC, despite lobar or main PVI. Nevertheless, all of these findings need to be validated in prospective clinical trials.

\section{Conflicts of Interest}

Conflict of interest relevant to this article was not reported.

\section{Acknowledgments}

This study was supported by the National Nuclear R\&D Program through a National Research Foundation of Korea (NRF) grant (2010-001854) funded by the Korean Ministry of Education, Science and technotech. 


\section{References}

1. Parkin DM, Bray F, Ferlay J, Pisani P. Global cancer statistics, 2002. CA Cancer J Clin. 2005;55:74-108.

2. Bruix J, Sherman M; American Association for the Study of Liver Diseases. Management of hepatocellular carcinoma: an update. Hepatology. 2011;53:1020-2.

3. Fleissig A, Jenkins V, Catt S, Fallowfield L. Multidisciplinary teams in cancer care: are they effective in the UK? Lancet Oncol. 2006;7:935-43.

4. Fennell ML, Das IP, Clauser S, Petrelli N, Salner A. The organization of multidisciplinary care teams: modeling internal and external influences on cancer care quality. J Natl Cancer Inst Monogr. 2010;2010:72-80.

5. Zaorsky NG, Trabulsi EJ, Lin J, Den RB. Multimodality therapy for patients with high-risk prostate cancer: current status and future directions. Semin Oncol. 2013;40:308-21.

6. Hadziahmetovic M, Shirai K, Chakravarti A. Recent advancements in multimodality treatment of gliomas. Future Oncol. 2011;7:1169-83.

7. Stillwagon GB, Order SE, Guse C, Klein JL, Leichner PK, Leibel SA, et al. 194 hepatocellular cancers treated by radiation and chemotherapy combinations: toxicity and response: a Radiation Therapy Oncology Group Study. Int J Radiat Oncol Biol Phys. 1989;17:1223-9.

8. Mornex F, Girard N, Beziat C, Kubas A, Khodri M, Trepo C, et al. Feasibility and efficacy of high-dose three-dimensionalconformal radiotherapy in cirrhotic patients with small-size hepatocellular carcinoma non-eligible for curative therapies-mature results of the French Phase II RTF-1 trial. Int J Radiat Oncol Biol Phys. 2006;66:1152-8.

9. Seong J, Lee IJ, Shim SJ, Lim DH, Kim TH, Kim JH, et al. A multicenter retrospective cohort study of practice patterns and clinical outcome on radiotherapy for hepatocellular carcinoma in Korea. Liver Int. 2009;29:147-52.

10. Yoon HI, Lee IJ, Han KH, Seong J. Improved oncologic outcomes with image-guided intensity-modulated radiation therapy using helical tomotherapy in locally advanced hepatocellular carcinoma. J Cancer Res Clin Oncol. 2014;140:1595-605.

11. National Comprehesive Cancer Network. NCCN clinical practice guidelines in oncology, hepatobiliary cancers [Internet]. Fort Washington, MD: National Comprehensive Cancer Network; 2014 [cited 2015 Jan 2]. Available from: http:// www.nccn.org/professionals/physician_gls/pdf/hepatobiliary.pdf.

12. Han KH, Seong J, Kim JK, Ahn SH, Lee DY, Chon CY. Pilot clinical trial of localized concurrent chemoradiation therapy for locally advanced hepatocellular carcinoma with portal vein thrombosis. Cancer. 2008;113:995-1003.

13. Park MS, Kim SU, Park JY, Kim DY, Ahn SH, Han KH, et al. Combination treatment of localized concurrent chemoradiation therapy and transarterial chemoembolization in locally advanced hepatocellular carcinoma with intrahepatic metas- tasis. Cancer Chemother Pharmacol. 2013;71:165-73.

14. Ben-Josef E, Normolle D, Ensminger WD, Walker S, Tatro D, Ten Haken RK, et al. Phase II trial of high-dose conformal radiation therapy with concurrent hepatic artery floxuridine for unresectable intrahepatic malignancies. J Clin Oncol. 2005;23:8739-47.

15. Korean Liver Cancer Study Group. Report of randomized registration for hepatocellular carcinoma in Korea [Internet]. Seoul: Korean Liver Cancer Study Group; 2010 [cited 2015 Jan 1]. Available from: http://klcsg.or.kr/.

16. von Elm E, Altman DG, Egger M, Pocock SJ, Gotzsche PC, Vandenbroucke JP, et al. The Strengthening the Reporting of Observational Studies in Epidemiology (STROBE) statement: guidelines for reporting observational studies. Lancet. 2007; 370:1453-7.

17. Liver Cancer Study Group of Japan. The general rules for the clinical and pathological study of primary liver cancer. 4 th ed. Tokyo: Kanehara Co. Ltd.; 2000.

18. Park JW; Korean Liver Cancer Study Group and National Cancer Center. Practice guideline for diagnosis and treatment of hepatocellular carcinoma. Korean J Hepatol. 2004;10:88-98.

19. Llovet JM, Ricci S, Mazzaferro V, Hilgard P, Gane E, Blanc JF, et al. Sorafenib in advanced hepatocellular carcinoma. N Engl J Med. 2008;359:378-90.

20. Cheng AL, Kang YK, Chen Z, Tsao CJ, Qin S, Kim JS, et al. Efficacy and safety of sorafenib in patients in the Asia-Pacific region with advanced hepatocellular carcinoma: a phase III randomised, double-blind, placebo-controlled trial. Lancet Oncol. 2009;10:25-34.

21. Yoon SM, Lim YS, Won HJ, Kim JH, Kim KM, Lee HC, et al. Radiotherapy plus transarterial chemoembolization for hepatocellular carcinoma invading the portal vein: long-term patient outcomes. Int J Radiat Oncol Biol Phys. 2012;82:2004-11.

22. Yamada K, Izaki K, Sugimoto K, Mayahara H, Morita $Y$, Yoden $\mathrm{E}$, et al. Prospective trial of combined transcatheter arterial chemoembolization and three-dimensional conformal radiotherapy for portal vein tumor thrombus in patients with unresectable hepatocellular carcinoma. Int J Radiat Oncol Biol Phys. 2003;57:113-9.

23. Dawson LA, McGinn CJ, Normolle D, Ten Haken RK, Walker $\mathrm{S}$, Ensminger W, et al. Escalated focal liver radiation and concurrent hepatic artery fluorodeoxyuridine for unresectable intrahepatic malignancies. J Clin Oncol. 2000;18:2210-8.

24. Kim KH, Kim MS, Chang JS, Han KH, Kim DY, Seong J. Therapeutic benefit of radiotherapy in huge $(>/=10 \mathrm{~cm})$ unresectable hepatocellular carcinoma. Liver Int. 2014;34:784-94.

25. Lee HS, Choi GH, Choi JS, Kim KS, Han KH, Seong J, et al. Surgical resection after down-staging of locally advanced hepatocellular carcinoma by localized concurrent chemoradiotherapy. Ann Surg Oncol. 2014;21:3646-53. 